\title{
GENERALIZED HYPERGEOMETRIC BERNOULLI NUMBERS
}

\author{
KALYAN CHAKRABORTY AND TAKAO KOMATSU
}

\begin{abstract}
We introduce generalized hypergeometric Bernoulli numbers for Dirichlet characters. We study their properties, including relations, expressions and determinants. At the end in Appendix we derive first few expressions of these numbers.
\end{abstract}

\section{INTRODUCTION}

Let ${ }_{1} F_{1}(a ; b ; z)$ denote the confluent hypergeometric function defined by

$$
{ }_{1} F_{1}(a ; b ; z)=\sum_{n=0}^{\infty} \frac{(a)^{(n)}}{(b)^{(n)}} \frac{z^{n}}{n !}
$$

with the rising factorial $(x)^{(n)}=x(x+1) \ldots(x+n-1)(n \geq 1)$ and $(x)^{(0)}=1$. Also for $N \geq 1$, hypergeometric Bernoulli numbers $B_{N, n}([2,6,17,8,9,13,18])$ are given by

$$
\frac{1}{{ }_{1} F_{1}(1 ; N+1 ; t)}=\frac{t^{N} / N !}{e^{t}-\sum_{n=0}^{N-1} t^{n} / n !}=\sum_{n=0}^{\infty} B_{N, n} \frac{t^{n}}{n !}
$$

with $B_{1, n}=B_{n}$, the classical Bernoulli numbers, defined by

$$
\frac{t}{e^{t}-1}=\sum_{n=0}^{\infty} B_{n} \frac{t^{n}}{n !} .
$$

In [10] a result of Kamano on a multiple binomial sum is generalized, higher order hypergeometric Bernoulli polynomials are defined, and its differential equation and recursion formulas are studied among other properties. It is well-known that some of the known properties of the Bernoulli numbers can be obtained from fundamental relationships between complete and elementary symmetric functions. In [11] two new closed form representations for the Apostol-Bernoulli polynomials are derived as natural generalizations of those of the Bernoulli polynomials. In [17] an infinite family of relationships between complete and elementary symmetric functions is introduced. From these relationships, the author deduces connections between some lacunary recurrence relations with gaps of length $2 r$ for the Bernoulli numbers and the integer partitions into at most $r-1$ parts. In [20] new bounds for the ratio of two adjacent even-indexed Bernoulli numbers are obtained, solving Qi's conjecture on the related topic, getting a tighter lower bound for this ratio is obtained, and moreover, some conjectures on related topics are proposed.

In [14, hypergeometric Cauchy numbers are defined and studied as analog of the hypergeometric Bernoulli numbers. This concept is further generalized in [15] by introducing the general hypergeometric Cauchy polynomials. In [16], the classical Euler numbers are generalized to define the higher order hypergeometric Euler numbers. Recently, in [12, by using the Hasse-Teichmüller derivatives, two explicit expressions for the related numbers of higher order Appell polynomials are obtained. One of them presents a determinant expression for the related numbers of higher order Appell polynomials, which involves

2020 Mathematics Subject Classification. Primary 11B68; Secondary 11B37, 33C15.

Key words and phrases. Bernoulli numbers; hypergeometric Bernoulli numbers; hypergeometric functions; Dirichlet characters. 
several determinant expressions of special numbers, such as the higher order generalized hypergeometric Bernoulli and Cauchy numbers

In this paper, we introduce generalized hypergeometric Bernoulli numbers for Dirichlet characters. Let $\chi$ be a Dirichlet character with conductor $f$ and $N$ be a positive integer. We define the generalized hypergeometric Bernoulli numbers $B_{N, n, \chi}$ by

$$
\sum_{a=1}^{f} \frac{\chi(a) t^{N} e^{a t} / N !}{e^{f t}-\sum_{n=0}^{N-1} \frac{(f t)^{n}}{n !}}=\sum_{n=0}^{\infty} B_{N, n, \chi} \frac{t^{n}}{n !} \quad(|t|<2 \pi / f) .
$$

One recovers the classical generalized Bernoulli number (see, e.g., [1, 3, 4, 19]) $B_{n, \chi}:=$ $B_{1, n, \chi}$, which are simply Bernoulli numbers twisted by a Dirichlet character. As seen in [3. Chapter 4], generalized Bernoulli numbers and Bernoulli polynomials are related. A closed form expression for sums of products of any number of generalized Bernoulli numbers is given in [4] and shortened recurrence relations for generalized Bernoulli numbers and polynomials attached to a primitive Dirichlet character is being studied in [1].

\section{BASIC PROPERTIES}

We begin with some basic expressions of generalized hypergeometric Bernoulli numbers.

Proposition 1. Let $\chi=1$ be the trivial character of conductor 1 . For any $N \geq 1$ and $n \geq 0$, put $N(n):=\min \{N-1, n\}$. Then we have

$$
B_{N, n, \mathbf{1}}= \begin{cases}\sum_{m=0}^{N(n)}\left(\begin{array}{l}
n \\
m
\end{array}\right) B_{N, n-m} & \text { if } n \neq N, \\
1+\sum_{m=0}^{N-1}\left(\begin{array}{l}
N \\
m
\end{array}\right) B_{N, N-m} & \text { if } n=N .\end{cases}
$$

Especially, for $N=1$, we have

$$
B_{1, n, \mathbf{1}}= \begin{cases}B_{n} & \text { if } n \neq 1 \\ 1 / 2 & \text { if } n=1\end{cases}
$$

Proof. By the definition of generalized hypergeometric Bernoulli numbers, we have

$$
\begin{aligned}
\sum_{n=0}^{\infty} B_{N, n, 1} \frac{t^{n}}{n !} & =\frac{t^{N} e^{t} / N !}{e^{t}-\sum_{n=0}^{N-1} \frac{t^{n}}{n !}} \\
& =\frac{t^{N} / N !\left(e^{t}-\sum_{n=0}^{N-1} \frac{t^{n}}{n !}\right)+t^{N} / N ! \sum_{n=0}^{N-1} \frac{t^{n}}{n !}}{e^{t}-\sum_{n=0}^{N-1} \frac{t^{n}}{n !}} \\
& =\frac{t^{N}}{N !}+\sum_{n=0}^{N-1} \frac{t^{n}}{n !} \times \sum_{n=0}^{\infty} B_{N, n} \frac{t^{n}}{n !} \\
& =\frac{t^{N}}{N !}+\sum_{m=0}^{N-1} \sum_{n=0}^{\infty} B_{N, n} \frac{t^{m+n}}{m ! n !}
\end{aligned}
$$




$$
\begin{aligned}
& =\frac{t^{N}}{N !}+\sum_{\ell=0}^{N-1} \sum_{m=0}^{\ell} B_{N, \ell-m} \frac{t^{\ell}}{m !(\ell-m) !}+\sum_{\ell=N}^{\infty} \sum_{m=0}^{N-1} B_{N, \ell-m} \frac{t^{\ell}}{m !(\ell-m) !} \\
& =\sum_{\ell=0}^{N-1} \sum_{m=0}^{\ell}\left(\begin{array}{c}
\ell \\
m
\end{array}\right) B_{N, \ell-m} \frac{t^{\ell}}{\ell !}+\left(1+\sum_{m=0}^{N-1}\left(\begin{array}{c}
N \\
m
\end{array}\right) B_{N, N-m}\right) \frac{t^{N}}{N !} \\
& +\sum_{\ell=N+1}^{\infty} \sum_{m=0}^{N-1}\left(\begin{array}{c}
\ell \\
m
\end{array}\right) B_{N, \ell-m} \frac{t^{\ell}}{\ell !}
\end{aligned}
$$

and we get the assertion.

Howard [8, 9] defined the hypergeometric Bernoulli polynomials $B_{N, n}(x) \in \mathbb{Q}[x]$ by

$$
\frac{t^{N} e^{x t} / N !}{e^{t}-\sum_{n=0}^{N-1} \frac{t^{n}}{n !}}=\sum_{n=0}^{\infty} B_{N, n}(x) \frac{t^{n}}{n !} .
$$

Note that $B_{n}(x):=B_{1, n}(x)$ be the classical Bernoulli polynomial and $B_{N, n}(0)=B_{N, n}$. For classical case, the following relation between the generalized Bernoulli numbers and the Bernoulli polynomials is well-known [19, Proposition 4.1]:

$$
B_{n, \chi}=f^{n-1} \sum_{a=1}^{f} \chi(a) B_{n}\left(\frac{a}{f}\right) .
$$

The following proposition is a generalization of the above classical case.

Proposition 2. Let $\chi$ be a Dirichlet character of conductor $f$. For any $N \geq 1$ and $n \geq 0$,

$$
B_{N, n, \chi}=f^{n-N} \sum_{a=1}^{f} \chi(a) B_{N, n}\left(\frac{a}{f}\right) .
$$

Proof. By the definition (of hypergeometric Bernoulli polynomial), we have,

$$
\sum_{n=0}^{\infty} B_{N, n}\left(\frac{a}{f}\right) \frac{(f t)^{n}}{n !}=\frac{(f t)^{N} e^{a / f \times f t} / N !}{e^{f t}-\sum_{n=0}^{N-1} \frac{(f t)^{n}}{n !}} .
$$

Now multiplying by $\chi(a)$ and taking a sum on $a$ from 1 to $f$, we have

$$
\begin{aligned}
f^{n} \sum_{n=0}^{\infty} \sum_{a=1}^{f} \chi(a) B_{N, n}\left(\frac{a}{f}\right) \frac{t^{n}}{n !} & =f^{N} \sum_{a=1}^{f} \frac{\chi(a) t^{N} e^{a t} / N !}{e^{f t}-\sum_{n=0}^{N-1} \frac{(f t)^{n}}{n !}} \\
& =f^{N} \sum_{n=0}^{\infty} B_{N, n, \chi} \frac{t^{n}}{n !} .
\end{aligned}
$$

Therefore,

$$
B_{N, n, \chi}=f^{n-N} \sum_{a=1}^{f} \chi(a) B_{N, n}\left(\frac{a}{f}\right)
$$

for any $n \geq 0$.

Consequently, we can express the generalized hypergeometric Bernoulli numbers in terms of hypergeometric Bernoulli numbers 
Corollary 1. For any integers $N \geq 1$ and $n \geq 0$,

$$
B_{N, n, \chi}=\sum_{a=1}^{f} \chi(a) \sum_{k=0}^{n}\left(\begin{array}{l}
n \\
k
\end{array}\right) B_{N, k} a^{n-k} f^{k-N} .
$$

Proof. Since

$$
\begin{aligned}
\sum_{n=0}^{\infty} B_{N, n}(x) \frac{t^{n}}{n !} & =\frac{t^{N} e^{x t} / N !}{e^{t}-\sum_{n=0}^{N-1} \frac{t^{n}}{n !}} \\
& =\left(\sum_{n=0}^{\infty} B_{N, n} \frac{t^{n}}{n !}\right)\left(\sum_{m=0}^{\infty} x^{m} \frac{t^{m}}{m !}\right) \\
& =\sum_{n=0}^{\infty} \sum_{k=0}^{n}\left(\begin{array}{l}
n \\
k
\end{array}\right) B_{N, k} x^{n-k} \frac{t^{n}}{n !},
\end{aligned}
$$

comparing the coefficients on both sides, we get

$$
B_{N, n}(x)=\sum_{k=0}^{n}\left(\begin{array}{l}
n \\
k
\end{array}\right) B_{N, k} x^{n-k} .
$$

By Proposition 2 with $x=a / f$, we obtain the desired result.

\section{Fundamental properties of the generalized hypergeometric Bernoulli POLYNOMIALS}

We also define the generalized hypergeometric Bernoulli polynomials $B_{N, n, \chi}$ by

$$
\sum_{a=1}^{f} \frac{\chi(a) t^{N} e^{(x+a) t} / N !}{e^{f t}-\sum_{n=0}^{N-1} \frac{(f t)^{n}}{n !}}=\sum_{n=0}^{\infty} B_{N, n, \chi}(x) \frac{t^{n}}{n !} \quad(|t|<2 \pi / f) .
$$

When $N=1$ in (3.1), $B_{n, \chi}(x):=B_{1, n, \chi}(x)$ is the classical generalized Bernoulli polynomial. When $\chi=\mathbf{1}, B_{N, n}(x):=B_{N, n, \mathbf{1}}(x)$ is the hypergeometric Bernoulli polynomial in (2.1).

Proposition 3. Let $\chi=\mathbf{1}$ be the trivial character of conductor 1 . For any $N \geq 1$ and $n \geq 0$, we have

$$
B_{N, n, \mathbf{1}}(x)= \begin{cases}\sum_{i=0}^{n}\left(\begin{array}{c}
n \\
i
\end{array}\right) B_{N, n-i}(x) & \text { if } 0 \leq n \leq N-1, \\
\left(\begin{array}{c}
n \\
N
\end{array}\right) x^{n-N}+\sum_{i=0}^{N-1}\left(\begin{array}{c}
n \\
i
\end{array}\right) B_{N, n-i}(x) & \text { if } n \geq N .\end{cases}
$$

Especially, for $N=1$, we have

$$
B_{1, n, \mathbf{1}}(x)= \begin{cases}1 & \text { if } n=0 \\ n x^{n-1}+B_{n}(x) & \text { if } n \geq 1 .\end{cases}
$$

Proof. By the definition in (3.1), we have

$$
\begin{aligned}
& \sum_{n=0}^{\infty} B_{N, n, \mathbf{1}}(x) \frac{t^{n}}{n !}=\frac{t^{N} e^{(x+1) t} / N !}{e^{t}-\sum_{n=0}^{N-1} \frac{t^{n}}{n !}} \\
& =\frac{t^{N} / N !\left(e^{t}-\sum_{n=0}^{N-1} \frac{t^{n}}{n !}\right) e^{x t}+t^{N} / N ! e^{x t} \sum_{n=0}^{N-1} \frac{t^{n}}{n !}}{e^{t}-\sum_{n=0}^{N-1} \frac{t^{n}}{n !}}
\end{aligned}
$$




$$
\begin{aligned}
= & \frac{t^{N}}{N !} e^{x t}+\left(\sum_{n=0}^{N-1} \frac{t^{n}}{n !}\right)\left(\sum_{n=0}^{\infty} B_{N, n}(x) \frac{t^{n}}{n !}\right) \\
= & \sum_{n=0}^{\infty} \frac{x^{n}}{N ! n !} t^{N+n}+\sum_{n=0}^{\infty} \sum_{i=0}^{\min \{n, N-1\}}\left(\begin{array}{c}
n \\
i
\end{array}\right) B_{N, n-i}(x) \frac{t^{n}}{n !} \\
= & \sum_{n=N}^{\infty}\left(\begin{array}{c}
n \\
N
\end{array}\right) x^{n-N} \frac{t^{n}}{n !}+\sum_{n=0}^{N-1} \sum_{i=0}^{n}\left(\begin{array}{c}
n \\
i
\end{array}\right) B_{N, n-i}(x) \frac{t^{n}}{n !} \\
& +\sum_{n=N}^{\infty} \sum_{i=0}^{N-1}\left(\begin{array}{c}
n \\
i
\end{array}\right) B_{N, n-i}(x) \frac{t^{n}}{n !} .
\end{aligned}
$$

Comparing the coefficients on both sides, we get the result.

Proposition 4. For $n \geq 0$,

$$
B_{N, n, \chi}(x+y)=\sum_{k=0}^{n}\left(\begin{array}{l}
n \\
k
\end{array}\right) B_{N, k, \chi}(x) y^{n-k} .
$$

Proof. By the definition in (3.1), we have

$$
\begin{aligned}
\sum_{n=0}^{\infty} B_{N, n, \chi}(x+y) \frac{t^{n}}{n !} & =\left(\sum_{n=0}^{\infty} B_{N, n, \chi}(x) \frac{t^{n}}{n !}\right) e^{y t} \\
& =\left(\sum_{k=0}^{\infty} B_{N, k, \chi}(x) \frac{t^{k}}{k !}\right)\left(\sum_{l=0}^{\infty} \frac{y^{l} t^{l}}{l !}\right) \\
& =\sum_{n=0}^{\infty} \sum_{k=0}^{n}\left(\begin{array}{l}
n \\
k
\end{array}\right) B_{N, k, \chi}(x) y^{n-k} \frac{t^{n}}{n !} .
\end{aligned}
$$

Comparing the coefficients on both sides, we get the result.

When $x=0$ and $y$ is replaced by $x$ in Proposition 2, we have a relation between generalized hypergeometric Bernoulli polynomials and numbers.

Corollary 2. For $n \geq 0$,

$$
B_{N, n, \chi}(x)=\sum_{k=0}^{n}\left(\begin{array}{l}
n \\
k
\end{array}\right) B_{N, k, \chi} x^{n-k} .
$$

There is another relation of generalized hypergeometric Bernoulli polynomials with their numbers in terms of Stirling numbers of the second kind $\left\{\begin{array}{l}n \\ k\end{array}\right\}$, whose generating function is given by

$$
\frac{\left(e^{t}-1\right)^{\ell}}{\ell !}=\sum_{n=\ell}^{\infty}\left\{\begin{array}{l}
n \\
\ell
\end{array}\right\} \frac{t^{n}}{n !}
$$

Proposition 5. For $n \geq 0$,

$$
B_{N, n, \chi}(x)=\sum_{k=0}^{n} \sum_{l=0}^{k}\left(\begin{array}{l}
n \\
k
\end{array}\right)\left\{\begin{array}{l}
k \\
l
\end{array}\right\}(x)_{l} B_{N, n-k, \chi},
$$

where $(x)_{l}=x(x-1) \cdots(x-l+1)(l \geq 1)$ denotes the falling factorial with $(x)_{0}=1$. 
Proof.

$$
\begin{aligned}
\sum_{n=0}^{\infty} B_{N, n, \chi}(x) \frac{t^{n}}{n !} & =\left(\sum_{n=0}^{\infty} B_{N, n, \chi} \frac{t^{n}}{n !}\right)\left(\left(e^{t}-1\right)+1\right)^{x} \\
& =\left(\sum_{n=0}^{\infty} B_{N, n, \chi} \frac{t^{n}}{n !}\right)\left(\sum_{l=0}^{\infty}\left(\begin{array}{l}
x \\
l
\end{array}\right) l ! \sum_{n=l}^{\infty}\left\{\begin{array}{l}
n \\
l
\end{array}\right\} \frac{t^{n}}{n !}\right) \\
& =\left(\sum_{n=0}^{\infty} B_{N, n, \chi} \frac{t^{n}}{n !}\right)\left(\sum_{n=0}^{\infty} \sum_{l=0}^{n}\left\{\begin{array}{l}
n \\
l
\end{array}\right\}(x)_{l} \frac{t^{n}}{n !}\right) \\
& =\sum_{n=0}^{\infty} \sum_{k=0}^{n} \sum_{l=0}^{k}\left(\begin{array}{l}
n \\
k
\end{array}\right)\left\{\begin{array}{l}
k \\
l
\end{array}\right\}(x)_{l} B_{N, n-k, \chi} \frac{t^{n}}{n !} .
\end{aligned}
$$

Comparing the coefficients on both sides, we get the result.

We can see that the generalized hypergeometric Bernoulli numbers are Appell.

Proposition 6. For $n \geq 1$,

$$
\frac{d}{d x} B_{N, n, \chi}(x)=n B_{N, n-1, \chi}(x) .
$$

Proof. From the definition in (3.1),

$$
\begin{aligned}
\frac{d}{d x} \sum_{n=0}^{\infty} B_{N, n, \chi}(x) \frac{t^{n}}{n !} & =t \sum_{n=0}^{\infty} B_{N, n, \chi}(x) \frac{t^{n}}{n !} \\
& =\sum_{n=0}^{\infty}(n+1) B_{N, n, \chi}(x) \frac{t^{n+1}}{(n+1) !} \\
& =\sum_{n=1}^{\infty} n B_{N, n-1, \chi}(x) \frac{t^{n}}{n !} .
\end{aligned}
$$

Comparing the coefficients on both sides, we get the result.

\section{Recurrence Relations}

From now on, for convenience sake let us put

$$
S_{n}:=\sum_{a=1}^{f} \chi(a) a^{n} \quad \text { and } \quad S_{n}(x):=\sum_{a=1}^{f} \chi(a)(x+a)^{n} .
$$

The following are recurrence relations of the generalized hypergeometric Bernoulli numbers and polynomials with Dirichlet character.

Proposition 7. For $n \geq 1$,

$$
\begin{array}{r}
\left(\begin{array}{c}
N+n \\
n
\end{array}\right) \sum_{i=0}^{-1}\left(\begin{array}{c}
N+n \\
i
\end{array}\right) B_{N, i, \chi} f^{N+n-i}=S_{n}, \\
\left(\begin{array}{c}
N+n \\
n
\end{array}\right)^{-1} \sum_{i=0}^{n}\left(\begin{array}{c}
N+n \\
i
\end{array}\right) B_{N, i, \chi}(x) f^{N+n-i}=S_{n}(x) .
\end{array}
$$


Remark. When $N=1$ in Proposition 7, we have

$$
\begin{array}{r}
\sum_{i=0}^{n-1}\left(\begin{array}{c}
n \\
i
\end{array}\right) \frac{B_{i+1, \chi}}{i+1} f^{n-i}+\frac{B_{0, \chi}}{n+1} f^{n+1}=S_{n}, \\
\sum_{i=0}^{n-1}\left(\begin{array}{c}
n \\
i
\end{array}\right) \frac{B_{i+1, \chi}(x)}{i+1} f^{n-i}+\frac{B_{0, \chi}(x)}{n+1} f^{n+1}=S_{n}(x) .
\end{array}
$$

which are [1, (1.12),(1.13)]. When $N=f=1$ in Proposition 7 , we have the well-known basic recurrence relations

$$
\begin{array}{r}
\sum_{i=0}^{n-1}\left(\begin{array}{c}
n \\
i
\end{array}\right) \frac{B_{i+1}}{i+1}+\frac{1}{n+1}=0 ; \\
\sum_{i=0}^{n-1}\left(\begin{array}{c}
n \\
i
\end{array}\right) \frac{B_{i+1}(x)}{i+1}+\frac{1}{n+1}=x^{n} .
\end{array}
$$

When $\chi=1$ is the trivial character of conductor 1 , the relation (4.1) is reduced to

$$
\sum_{i=0}^{n}\left(\begin{array}{c}
N+n \\
i
\end{array}\right) B_{N, i, \mathbf{1}}=0 \text {. }
$$

which is [2, Proposition $1(3)]$.

Proof of Proposition 7. We shall prove the relation (4.2). The relation (4.1) is the special case when $x=0$. From the definition in (3.1),

$$
\sum_{a=1}^{f} \chi(a) \frac{t^{N}}{N !} e^{(x+a) t}=\left(e^{f t}-\sum_{n=0}^{N-1} \frac{(f t)^{n}}{n !}\right)\left(\sum_{n=0}^{\infty} B_{N, n, \chi}(x) \frac{t^{n}}{n !}\right) .
$$

The right-hand side is equal to

$$
\left(\sum_{n=0}^{\infty} \frac{(f t)^{N+n}}{(N+n) !}\right)\left(\sum_{n=0}^{\infty} B_{N, n, \chi}(x) \frac{t^{n}}{n !}\right)=\sum_{n=0}^{\infty} \sum_{i=0}^{n} \frac{B_{N, i, \chi}(x)}{i !} \frac{f^{N+n-i}}{(N+n-i) !} t^{N+n} .
$$

The left-hand side is equal to

$$
\sum_{n=0}^{\infty} \sum_{a=1}^{f} \chi(a) \frac{t^{N}}{N !}(x+a)^{n} \frac{t^{n}}{n !}=\sum_{n=0}^{\infty} \sum_{a=1}^{f} \chi(a)(x+a)^{n} \frac{t^{N+n}}{N ! n !} .
$$

Comparing the coefficients on both sides, we get

$$
\sum_{i=0}^{n} \frac{B_{N, i, \chi}(x)}{i !} \frac{f^{N+n-i}}{(N+n-i) !}=\sum_{a=1}^{f} \frac{\chi(a)(x+a)^{n}}{N ! n !}
$$

yielding the relation (4.2).

\section{EXPRESSIONS} bers.

We give expressions of the generalized hypergeometric Bernoulli polynomials and num-

Theorem 1. For $n \geq 1$,

$$
B_{N, n, \chi}=\sum_{k=0}^{n} \frac{k !}{f^{N-k}}\left(\begin{array}{l}
n \\
k
\end{array}\right) \sum_{r=0}^{k} T_{r}(k) S_{n-k}
$$




$$
B_{N, n, \chi}(x)=\sum_{k=0}^{n} \frac{k !}{f^{N-k}}\left(\begin{array}{l}
n \\
k
\end{array}\right) \sum_{r=0}^{k} T_{r}(k) S_{n-k}(x) .
$$

where

$$
T_{r}(k):=(-N !)^{r} \sum_{\substack{i_{1}+\cdots+i_{r}=k \\ i_{1}, \ldots, i_{r} \geq 1}} \frac{1}{\left(N+i_{1}\right) ! \cdots\left(N+i_{r}\right) !}
$$

with $T_{0}(0)=1$ and $T_{0}(k)=0(k \geq 1)$.

Remark. When $\chi=\mathbf{1}$ is the trivial character of conductor 1, expression (5.1) reduces to

$$
B_{N, n, \mathbf{1}}=n ! \sum_{r=1}^{n}(-N !)^{r} \sum_{\substack{i_{1}+\cdots+i_{r}=n \\ i_{1}, \ldots, i_{r} \geq 1}} \frac{1}{\left(N+i_{1}\right) ! \cdots\left(N+i_{r}\right) !},
$$

which is [2, Proposition 1 (4)].

Proof of Theorem 1. We shall prove (5.2) as (5.1) can be easily deduced. From the definition in (3.1),

$$
\begin{aligned}
& \sum_{n=0}^{\infty} B_{N, n, \chi}(x) \frac{t^{n}}{n !} \\
& =\left(\sum_{a=1}^{f} \chi(a) \sum_{n=0}^{\infty}(x+a)^{n} \frac{t^{n}}{n !}\right) \frac{1}{f^{N}}\left(\sum_{n=0}^{\infty} \frac{N ! f^{n}}{(N+n) !} t^{n}\right)^{-1} \\
& =\frac{1}{f^{N}}\left(\sum_{n=0}^{\infty} S_{n}(x) \frac{t^{n}}{n !}\right) \sum_{r=0}^{\infty}\left(-\sum_{n=1}^{\infty} \frac{N ! f^{n}}{(N+n) !} t^{n}\right)^{r} \\
& =\frac{1}{f^{N}}\left(\sum_{n=0}^{\infty} S_{n}(x) \frac{t^{n}}{n !}\right) \sum_{r=0}^{\infty}(-1)^{r} \sum_{n=r}^{\infty} \sum_{\substack{i_{1}+\cdots+i_{r}=n \\
i_{1}, \ldots, i_{r} \geq 1}} \frac{(N !)^{r} f^{n}}{\left(N+i_{1}\right) ! \cdots\left(N+i_{r}\right) !} t^{n} \\
& =\frac{1}{f^{N}}\left(\sum_{n=0}^{\infty} S_{n}(x) \frac{t^{n}}{n !}\right) \sum_{n=0}^{\infty} \sum_{r=0}^{n}(-N !)^{r} \sum_{\substack{i_{1}+\cdots, i_{r}=n \\
i_{1}, \ldots, i_{r} \geq 1}} \frac{n ! f^{n}}{\left(N+i_{1}\right) ! \cdots\left(N+i_{r}\right) !} \frac{t^{n}}{n !} \\
& =\frac{1}{f^{N}} \sum_{n=0}^{\infty} \sum_{k=0}^{n}\left(\begin{array}{l}
n \\
k
\end{array}\right) S_{n-k}(x) \sum_{r=0}^{k}(-N !)^{r} \sum_{\substack{i_{1}+\cdots, i_{r}=k \\
i_{1}, \ldots, i_{r} \geq 1}} \frac{k ! f^{k}}{\left(N+i_{1}\right) ! \cdots\left(N+i_{r}\right) !} \frac{t^{n}}{n !} .
\end{aligned}
$$

Comparing the coefficients on both sides, we get (5.2). Note that the summation is empty when $k \geq 1$ and $r=0$.

There are alternative expressions with additional binomial coefficients.

Theorem 2. For $n \geq 1$,

$$
\begin{gathered}
B_{N, n, \chi}=\sum_{k=0}^{n} \frac{k !}{f^{N-k}}\left(\begin{array}{l}
n \\
k
\end{array}\right) \sum_{r=0}^{k}\left(\begin{array}{l}
k+1 \\
r+1
\end{array}\right) \widetilde{T}_{r}(k) S_{n-k}, \\
B_{N, n, \chi}(x)=\sum_{k=0}^{n} \frac{k !}{f^{N-k}}\left(\begin{array}{l}
n \\
k
\end{array}\right) \sum_{r=0}^{k}\left(\begin{array}{l}
k+1 \\
r+1
\end{array}\right) \widetilde{T}_{r}(k) S_{n-k}(x) .
\end{gathered}
$$

where

$$
\widetilde{T}_{r}(k):=(-N !)^{r} \sum_{\substack{i_{1}+\cdots+i_{r}=k \\ i_{1}, \ldots, i_{r} \geq 0}} \frac{1}{\left(N+i_{1}\right) ! \cdots\left(N+i_{r}\right) !}
$$


with $\widetilde{T}_{0}(0)=1$ and $\widetilde{T}_{0}(k)=0(k \geq 1)$.

Remark. When $\chi=\mathbf{1}$ is the trivial character of conductor 1 , (5.3) is reduced to

$$
B_{N, n, \mathbf{1}}=n ! \sum_{r=1}^{n}\left(\begin{array}{c}
n+1 \\
r+1
\end{array}\right)(-N !)^{r} \sum_{\substack{i_{1}+\cdots+i_{r}=n \\
i_{1}, \ldots, i_{r} \geq 0}} \frac{1}{\left(N+i_{1}\right) ! \cdots\left(N+i_{r}\right) !},
$$

which is [2, Proposition 1 (5)].

Proof of Theorem [2. We shall prove the expression (5.4). Put

$$
w:=\sum_{n=1}^{\infty} \frac{N ! f^{n}}{(N+n) !} t^{n} .
$$

Then, we have

$$
\begin{aligned}
& B_{N, n, \chi}(x):=\left.\frac{d^{n}}{d t^{n}} \frac{1}{f^{N}}(1+w)^{-1} \sum_{a=1}^{f} \chi(a) e^{(x+a) t}\right|_{t=0} \\
& =\left.\left.\frac{1}{f^{N}} \sum_{k=0}^{n}\left(\begin{array}{l}
n \\
k
\end{array}\right) \frac{d^{k}}{d t^{k}} \sum_{l=0}^{\infty}(-w)^{l}\right|_{t=0} \frac{d^{n-k}}{d t^{n-k}} \sum_{\ell=0}^{\infty} S_{\ell}(x) \frac{t^{\ell}}{\ell !}\right|_{t=0} \\
& =\frac{1}{f^{N}} \sum_{k=0}^{n}\left(\begin{array}{l}
n \\
k
\end{array}\right) \sum_{l=0}^{k} \sum_{r=0}^{l}(-1)^{r}\left(\begin{array}{l}
l \\
r
\end{array}\right) \\
& \times\left.\left.\frac{d^{k}}{d t^{k}}\left(\sum_{m=0}^{\infty} \frac{N ! f^{m}}{(N+m) !} t^{m}\right)^{r}\right|_{t=0} ^{\infty} \sum_{\ell=0}^{\infty} S_{n-k+\ell}(x) \frac{t^{\ell}}{\ell !}\right|_{t=0} \\
& =\frac{1}{f^{N}} \sum_{k=0}^{n}\left(\begin{array}{l}
n \\
k
\end{array}\right) \sum_{l=0}^{k} \sum_{r=0}^{l}(-1)^{r}\left(\begin{array}{l}
l \\
r
\end{array}\right) \\
& \times\left.\frac{d^{k}}{d t^{k}} \sum_{m=0}^{\infty} \sum_{\substack{i_{1}+\cdots+i_{r}=m \\
i_{1}, \ldots, i_{r} \geq 0}} \frac{(N !)^{r} f^{m}}{\left(N+i_{1}\right) ! \cdots\left(N+i_{r}\right) !} t^{m}\right|_{t=0} S_{n-k}(x) \\
& =\left.\frac{1}{f^{N}} \sum_{k=0}^{n}\left(\begin{array}{l}
n \\
k
\end{array}\right) \sum_{l=0}^{k} \sum_{r=0}^{l}\left(\begin{array}{l}
l \\
r
\end{array}\right) \sum_{m=k}^{\infty} \frac{m ! f^{m}}{(m-k) !} \widetilde{T}_{r}(m) t^{m-k}\right|_{t=0} S_{n-k}(x) \\
& =\frac{1}{f^{N}} \sum_{k=0}^{n}\left(\begin{array}{l}
n \\
k
\end{array}\right) \sum_{l=0}^{k} \sum_{r=0}^{l}\left(\begin{array}{l}
l \\
r
\end{array}\right) k ! f^{k} \widetilde{T}_{r}(k) S_{n-k}(x) \\
& =\sum_{k=0}^{n} \frac{k !}{f^{N-k}}\left(\begin{array}{l}
n \\
k
\end{array}\right) \sum_{r=0}^{k}\left(\sum_{l=r}^{k}\left(\begin{array}{l}
l \\
r
\end{array}\right)\right) \widetilde{T}_{r}(k) S_{n-k}(x) \\
& =\sum_{k=0}^{n} \frac{k !}{f^{N-k}}\left(\begin{array}{l}
n \\
k
\end{array}\right) \sum_{r=0}^{k}\left(\begin{array}{l}
k+1 \\
r+1
\end{array}\right) \widetilde{T}_{r}(k) S_{n-k}(x) \text {. }
\end{aligned}
$$

\section{Determinants}

Recurrence formula from Proposition 7

$$
B_{N, n, \chi}=\frac{S_{n}}{f^{N}}-\sum_{i=0}^{n-1} \frac{N ! n ! f^{n-i}}{(N+n-i) ! i !} B_{N, i, \chi}
$$


assists us to derive determinant expressions of generalized hypergeometric Bernoulli numbers and polynomials. Such determinant expressions were studied by Glaisher ([5]) extensively for Bernoulli, Cauchy, Euler and more numbers.

Theorem 3. For $n \geq 1$,

$$
B_{N, n, \chi}=(-1)^{n} n !\left|\begin{array}{ccccc}
\frac{N !}{(N+1) !} f & 1 & 0 & & \\
\frac{N !}{(N+2) !} f^{2} & \frac{N !}{(N+1) !} f & & & \\
\vdots & & & & 0 \\
\frac{N !}{(N+n-1) !} f^{n-1} & \ldots & \frac{N !}{(N+2) !} f^{2} & \frac{N !}{(N+1) !} f & 1 \\
\widehat{S}_{n} & \widehat{S}_{n-1} & \cdots & \widehat{S}_{2} & \widehat{S}_{1}
\end{array}\right|
$$

where

$$
\widehat{S}_{n}=\frac{1}{f^{N}} \sum_{a=1}^{f} \chi(a)\left(\frac{N !}{(N+n) !} f^{n}-\frac{a^{n}}{n !}\right) .
$$

Also

$$
B_{N, n, \chi}(x)=(-1)^{n} n !\left|\begin{array}{ccccc}
\frac{N !}{(N+1) !} f & 1 & 0 & & \\
\frac{N !}{(N+2) !} f^{2} & \frac{N !}{(N+1) !} f & & & \\
\vdots & & & & 0 \\
\frac{N !}{(N+n-1) !} f^{n-1} & \ldots & \frac{N !}{(N+2) !} f^{2} & \frac{N !}{(N+1) !} f & 1 \\
\widehat{S}_{n}(x) & \widehat{S}_{n-1}(x) & \cdots & \widehat{S}_{2}(x) & \widehat{S}_{1}(x)
\end{array}\right|
$$

where

$$
\widehat{S}_{n}(x)=\frac{1}{f^{N}} \sum_{a=1}^{f} \chi(a)\left(\frac{N !}{(N+n) !} f^{n}-\frac{(x+a)^{n}}{n !}\right) .
$$

Remark. When $\chi=\mathbf{1}$ is the trivial character of conductor 1 , by $\widehat{S}_{n}=N ! /(N+n)$ !, the determinant (6.2) is reduced to

$$
B_{N, n, \mathbf{1}}=(-1)^{n} n !\left|\begin{array}{ccccc}
\frac{N !}{(N+1) !} & 1 & 0 & & \\
\frac{N !}{(N+2) !} & \frac{N !}{(N+1) !} & & & \\
\vdots & & & & 0 \\
\frac{N !}{(N+n-1) !} & \cdots & \frac{N !}{(N+2) !} & \frac{N !}{(N+1) !} & 1 \\
\frac{N !}{(N+n) !} & \frac{N !}{(N+n-1) !} & \cdots & \frac{N !}{(N+2) !} & \frac{N !}{(N+1) !}
\end{array}\right|
$$

which is [2, Theorem 1].

Proof of Theorem 3. For simplicity, let

$$
\alpha_{i}=\frac{N ! f^{i}}{(N+i) !} \quad(i \geq 1),
$$

then

$$
\widehat{S}_{n}=\alpha_{n} \frac{S_{0}}{f^{N}}-\frac{S_{n}}{f^{N} n !}=\frac{1}{f_{N}}\left(\alpha_{n} S_{0}-\frac{S_{n}}{n !}\right) .
$$

Furthermore, by putting $\beta_{n}=(-1)^{n} B_{N, n, \chi} / n$ !, we shall show that

$$
\beta_{n}=\left|\begin{array}{ccccc}
\alpha_{1} & 1 & 0 & & \\
\alpha_{2} & \alpha_{1} & & & \\
\vdots & & & & 0 \\
\alpha_{n-1} & \cdots & \alpha_{2} & \alpha_{1} & 1 \\
\widehat{S}_{n} & \widehat{S}_{n-1} & \cdots & \widehat{S}_{2} & \widehat{S}_{1}
\end{array}\right|
$$


by using the recurrence relation

$$
\beta_{n}=\frac{(-1)^{n} S_{n}}{f^{N} n !}-\sum_{i=0}^{n-1}(-1)^{n-i} \alpha_{n-i} \beta_{i} \quad(n \geq 1) .
$$

By definition,

For $n=1$, by (6.5),

$$
\beta_{0}=B_{N, 0, \chi}=\frac{S_{0}}{f^{N}}
$$

$$
\beta_{1}=\alpha_{1} \beta_{0}-\frac{S_{1}}{f^{N}}=\widehat{S}_{1} .
$$

Hence (6.4) is valid. Assume that (6.4) is valid up to $n-1$. Then by expanding the determinant along the first row repeatedly, the right-hand side of (6.4) is equal to

$$
\begin{aligned}
& \alpha_{1} \beta_{n-1}-\left|\begin{array}{ccccc}
\alpha_{2} & 1 & 0 & \\
\alpha_{3} & \alpha_{1} & & \\
\vdots & \vdots & & & 0 \\
\alpha_{n-1} & \alpha_{n-3} & \cdots & \alpha_{1} & 1 \\
\widehat{S}_{n} & \widehat{S}_{n-2} & \cdots & \widehat{S}_{2} & \widehat{S}_{1}
\end{array}\right| \\
& =\alpha_{1} \beta_{n-1}-\alpha_{2} \beta_{n-2}+\cdots+(-1)^{n-1} \alpha_{n-2} \beta_{2}+(-1)^{n}\left|\begin{array}{cc}
\alpha_{n-1} & 1 \\
\widehat{S}_{n} & \widehat{S}_{1}
\end{array}\right| \\
& =\alpha_{1} \beta_{n-1}-\alpha_{2} \beta_{n-2}+\cdots+(-1)^{n-1} \alpha_{n-2} \beta_{2}+(-1)^{n} \alpha_{n-1} \beta_{1}+(-1)^{n+1} \widehat{S}_{n} \\
& =\beta_{n} .
\end{aligned}
$$

The last equality is due to the recurrence relation (6.5). Then the first relation (6.2) is proved. The second equation (6.3) can be proved similarly, by using the recurrence relation

$$
\frac{B_{N, n, \chi}(x)}{n !}=\frac{S_{n}(x)}{f^{N} n !}-\sum_{i=0}^{n-1} \frac{N ! f^{n-i}}{(N+n-i) !} \frac{B_{N, i, \chi}(x)}{i !}
$$

or

$$
\beta_{n}(x)=\frac{(-1)^{n} S_{n}(x)}{f^{N} n !}-\sum_{i=0}^{n-1}(-1)^{n-i} \alpha_{n-i} \beta_{i}(x) \quad(n \geq 1) .
$$

\section{ACKNOWLEDGEMENTS}

The authors are grateful to three anonymous referees for their precious comments and advices. This work has been partly done when the second author (T.K.) visited HarishChandra Research Institute in February 2020, where the first author (K. C.) was working before moving to his current institute. T. K. thanks K. C. for the warm hospitality of the institute. This work was initiated from a hint by Professor Miho Aoki of Shimane University. Both authors thank her.

\section{REFERENCES}

[1] T. Agoh, Shortened recurrence relations for generalized Bernoulli numbers and polynomials, J. Number Theory 176 (2017), 149-173.

[2] M. Aoki, T. Komatsu and G. K. Panda, Several properties of hypergeometric Bernoulli numbers, J. Inequal. Appl. 2019 (2019), Paper No.113. 24p.

[3] T. Arakawa, T. Ibukiyama and M. Kaneko, Bernoulli numbers and zeta functions. With an appendix by Don Zagier, Springer Monographs in Mathematics, Springer, Tokyo, 2014.

[4] K.-W. Chen and M. Eie, A note on generalized Bernoulli numbers, Pacific J. Math. 199 (2001), 41-59.

[5] J. W. L. Glaisher, Expressions for Laplace's coefficients, Bernoullian and Eulerian numbers etc. as determinants, Messenger (2) 6 (1875), 49-63. 
[6] A. Hassen and H. D. Nguyen, Hypergeometric Bernoulli polynomials and Appell sequences, Int. J. Number Theory 4 (2008), 767-774.

[7] A. Hassen and H. D. Nguyen, Hypergeometric zeta functions, Int. J. Number Theory 6 (2010), 99-126.

[8] F. T. Howard, A sequence of numbers related to the exponential function, Duke Math. J. 34 (1967), 599-615.

[9] F. T. Howard, Some sequences of rational numbers related to the exponential function, Duke Math. J. 34 (1967), 701-716.

[10] S. Hu and M.-S. Kim, On hypergeometric Bernoulli numbers and polynomials, Acta Math. Hungar. 154(1) (2018), 134-146.

[11] S. Hu and M.-S. Kim, Two closed forms for the Apostol-Bernoulli polynomials, Ramanujan J. 46 (2018), 103-117.

[12] S. Hu and T. Komatsu, Explicit expressions for the related numbers of higher order Appell polynomials, Quaest. Math. 43(8) (2020), 1019-1029.

[13] K. Kamano, Sums of products of hypergeometric Bernoulli numbers, J. Number Theory 130 (2010), 2259-2271.

[14] T. Komatsu, Hypergeometric Cauchy numbers, Int. J. Number Theory 9 (2013), 545-560.

[15] T. Komatsu and P. Yuan, Hypergeometric Cauchy numbers and polynomials, Acta Math. Hungar. 153 (2017), 382-400.

[16] T. Komatsu and W. Zhang, Several properties of multiple hypergeometric Euler numbers, Tokyo J. Math. 42(2) (2019), 551-570.

[17] M. Merca, Bernoulli numbers and symmetric functions, Rev. R. Acad. Cienc. Exactas Fis. Nat. Ser. A Mat. RACSAM 114(1) (2020), Paper No.20, 16 pp.

[18] H. D. Nguyen, Sums of products of hypergeometric Bernoulli polynomials, MAA-NJ Section - Spring Meeting, Middle Country College, NJ, April 10, 2010.

[19] L. C. Washington, Introduction to cyclotomic fields, second edition, GTM 83, Springer-Verlag, New York, 1997.

[20] L. Zhu, New bounds for the ratio of two adjacent even-indexed Bernoulli numbers, Rev. R. Acad. Cienc. Exactas Fis. Nat. Ser. A Mat. RACSAM 114(2) (2020), Paper No. 83, 13 pp.

\section{APPENDIX}

We derive first few values of $B_{N, n, \chi}$ :

$$
\begin{aligned}
B_{N, 0, \chi}= & \frac{S_{0}}{f^{N}}, \\
B_{N, 1, \chi}= & -\frac{S_{0}}{(N+1) f^{N-1}}+\frac{S_{1}}{f^{N}}, \\
B_{N, 2, \chi}= & \frac{2 S_{0}}{(N+1)^{2}(N+2) f^{N-2}}-\frac{2 S_{1}}{(N+1) f^{N-1}}+\frac{S_{2}}{f^{N}}, \\
B_{N, 3, \chi}= & \frac{3 !(N-1) S_{0}}{(N+1)^{3}(N+2)(N+3) f^{N-3}}+\frac{3 ! S_{1}}{(N+1)^{2}(N+2) f^{N-2}}-\frac{3 S_{2}}{(N+1) f^{N-1}}+\frac{S_{3}}{f^{N}}, \\
B_{N, 4, \chi}= & \frac{4 !\left(N^{3}-N^{2}-6 N+2\right) S_{0}}{(N+1)^{4}(N+2)^{2}(N+3)(N+4) f^{N-4}}+\frac{4 !(N-1) S_{1}}{(N+1)^{3}(N+2)(N+3) f^{N-3}} \\
& \quad+\frac{12 S_{2}}{(N+1)^{2}(N+2) f^{N-2}}-\frac{4 S_{3}}{(N+1) f^{N-1}}+\frac{S_{4}}{f^{N}} .
\end{aligned}
$$

Now

$$
\begin{aligned}
& B_{N, 0}=1 \\
& B_{N, 1}=-\frac{1}{N+1}, \\
& B_{N, 2}=\frac{2}{(N+1)^{2}(N+2)}, \\
& B_{N, 3}=\frac{3 !(N-1)}{(N+1)^{3}(N+2)(N+3)},
\end{aligned}
$$




$$
B_{N, 4}=\frac{4 !\left(N^{3}-N^{2}-6 N+2\right)}{(N+1)^{4}(N+2)^{2}(N+3)(N+4)} .
$$

From ([2]) we can see that,

$$
B_{N, n, \chi}=\sum_{k=0}^{n}\left(\begin{array}{l}
n \\
k
\end{array}\right) \frac{S_{n-k} B_{N, k}}{f^{N-k}}
$$

as shown in Corollary 1 .

When $N=1$ and $\chi$ is not the trivial character, as $S_{0}=0$, we find that

$$
\begin{aligned}
& B_{1,0, \chi}=0 \\
& B_{1,1, \chi}=\frac{S_{1}}{f}, \\
& B_{1,2, \chi}=-S_{1}+\frac{S_{2}}{f}, \\
& B_{1,3, \chi}=\frac{f S_{1}}{2}-\frac{3 S_{2}}{2}+\frac{S_{3}}{f}, \\
& B_{1,4, \chi}=f S_{2}-2 S_{3}+\frac{S_{4}}{f} .
\end{aligned}
$$

Harish-Chandra Research Institute, Allahabad, 211019, Uttar Pradesh, India, Kerala school of Mathematics, Kunnamangalam, Kozhikode, 673571, Kerala, India

Email address: kalyan@hri.res.in, kalychak@ksom.res.in

Department of Mathematical Sciences, School of Science, Zhejiang Sci-Tech University, HANGZHOU 310018, China

Email address: komatsu@zstu.edu.cn 